\title{
Enseñanza superior y sociedad: un estudio exploratorio sobre prácticas de la tercera misión en la Universidade Estadual de Campinas (Unicamp)
}

\author{
Ana Maria Nunes Gimenez ${ }^{1^{*}}$, Maria Beatriz Machado Bonacelli ${ }^{2}$
}

\begin{abstract}
Resumen: Este artículo presenta los resultados del estudio realizado sobre la tercera misión en la Universidade Estadual de Campinas (Unicamp), así como presenta un esbozo conceptual de la tercera misión, con base en estudios de referencia en ese campo, especialmente enfatizando los enfoques que posibilitan una visión multidimensional de la relación universidad-sociedad. Se utilizaron fuentes primarias (entrevistas y cuestionarios) y secundarias (informes institucionales, anuarios estadísticos y bibliografía especializada). Se concluye que la Unicamp está involucrada en diversas actividades de la tercera misión, sin embargo, los resultados también indican que la falta de comunicación y de intercambio de informaciones, las visiones estrechas de la relación universidad-sociedad y las reducidas concepciones de la extensión universitaria y de la tercera misión están entre las realidades que limitan e impiden el establecimiento de una comprensión holística de la tercera misión.
\end{abstract}

Palabras clave: tercera misión; extensión; desarrollo regional; compromiso social; transferencia de tecnología.

\begin{abstract}
Higher education and society: an exploratory study on practices of the third mission at the University of Campinas (Unicamp)
This article presents the partial results of a study that investigated the third mission at the University of Campinas (Unicamp), as well as a conceptual outline of the third mission, based on reference studies in this field, especially emphasizing the approaches that would allow a multidimensional view of the university-society relationship. We used primary sources (interviews and questionnaires) and secondary sources (institutional reports, statistical yearbooks and specialized bibliography). It is concluded that Unicamp is engaged in several activities of the third mission, however, the results also indicate that the lack of communication and exchange of information, the close visions of the university-society relationship and the narrow conceptions of university extension and the third mission are among the realities that limit and hinder the establishment of a holistic understanding of the third mission.
\end{abstract}

Keywords: third mission; extension; regional development; social engagement; technology transfer

Submitted: August $5^{\text {th }}, 2018 /$ Approved: November $21^{\text {st }}, 2018$

\section{Introducción}

Cada vez más se entiende que las instituciones de enseñanza superior (IES) y, especialmente, las universidades intensivas en investigación, deben estrechar los vínculos con la sociedad asumiendo mayor visibilidad, prestando servicios, promoviendo el emprendedorismo y la innovación y contribuyendo más activamente al desarrollo socioeconómico local/regional. De este modo, en el siglo XXI, se exige cada vez más que las IES extiendan y refuercen sus acciones más allá de sus muros, considerando todos los posibles impactos que esas acciones puedan promover - sobre la comunidad, sobre las empresas, sobre los gobiernos locales, sobre las instituciones y organizaciones de su entorno. Este conjunto de relaciones ha sido considerado como integrante de la tercera misión (siendo que la primera es la enseñanza y la segunda es la investigación).

Lo que se nota es que ha sido bastante común asociar la tercera misión directamente con actividades de capitalización de conocimiento, emprendedorismo, innovación y transferencia de tecnología. Por otro lado, todas las actividades ajenas a esas relaciones, pero que también involucran la relación universidad-sociedad suelen ser nombradas de extensión (actividades culturales, cursos, proyectos sociales y otros). Sin embargo, diversos esfuerzos internacionales han contribuido a la evolución del entendimiento de la relación universidad-sociedad a partir de la creación de un cuerpo de conocimientos en ese campo, apuntando a la urgencia de un tratamiento más amplio, que se libere de esa visión polarizada. Estos estudios sostienen que la "tercera misión" representa un conjunto amplio de interacciones de la universidad con la sociedad, según lo presentado por Molas-Gallart, Salter, Patel, Scott y Duran (2002); Schoen (2006); Grao, Iriarte, Ochoa y Vieira (2014); European Indicators and Ranking Methodology for University Third Mission - E3M (2012); D’Este, Castro-Martínez y Molas-Gallart (2014), Mora y Vieira (2014) y otros.

Por lo tanto, el estudio se justifica porque se nota que, aunque la comprensión del papel de la universidad ha evolucionado, la idea de tercera misión todavía suscita muchas discusiones, no sólo desde el punto de vista conceptual, sino principalmente en lo que se refiere a su alcance, es decir, a los tipos de actividades y relaciones abarcadas por el concepto. En vista de estas evidencias, el presente artículo fue estructurado para que fuera posible presentar los resultados parciales de la investigación conducida a nivel de doctorado, que investigó las múltiples caras de la relación universidad-sociedad, o sea, de la realización de la tercera misión en la Universidade Estadual de Campinas (Unicamp).

(1) Departamento de Política Científica y Tecnológica, Instituto de Geociencias, Universidade Estadual de Campinas.

(2) Programa de Postgrado en Política Científica y Tecnológica, Instituto de Geociencias, Universidade Estadual de Campinas.

*Corresponding author: anamarianunesgimenez@gmail.com

ISSN: 0718-2724. (http://jotmi.org)

Journal of Technology Management \& Innovation @ Universidad Alberto Hurtado, Facultad de Economía y Negocios. 
Esta investigación tuvo fines exploratorios y descriptivos y el abordaje adoptado fue el cualitativo. Además de las fuentes bibliográficas que estructuraron el referencial teórico-conceptual, también se utilizaron las siguientes fuentes: documentos institucionales - anuarios estadísticos; informes de actividades; con vistas a la identificación de las iniciativas de la universidad en ese campo; y también cuestionarios y entrevistas, para identificar los desafios y limitaciones para realización de la relación universidad-sociedad en la Unicamp. Además de esta introducción y de las conclusiones, el artículo está compuesto por otras tres partes que son las siguientes: marco teórico-conceptual, con una breve presentación de las misiones universitarias, especialmente la evolución de la tercera misión; procedimientos metodológicos, que aclara aspectos específicos de la recolección de los datos empíricos en la Unicamp; presentación y discusión de los resultados.

Los hallazgos de la investigación permitieron concluir que la Unicamp está activamente involucrada en la conducción de varias iniciativas de la tercera misión. Además de destacarse en el escenario nacional, especialmente en el campo del emprendedorismo, innovación y transferencia de tecnología. Sin embargo, todavía existen cuestiones que necesitan ser perfeccionadas, como la mejora de los canales de comunicación, la interacción y la promoción de sinergias entre las diversas instancias que promueven la relación universidad-sociedad, es decir, de la tercera misión de la universidad.

\section{Marco teórico}

\subsection{Universidad: origen y misiones}

Los diversos autores sostienen que, aunque ya existiese la enseñanza superior en Grecia, en Roma y en el mundo musulmán, fue sólo a partir del siglo XI que se puede hablar de un tipo de enseñanza muy próximo a lo que conocemos hoy: facultades, currículos (con definición de asuntos, programas y tiempo de estudios), exámenes, grados académicos, etc. y eso es una herencia directa, no de Atenas o de Alejandría, sino de París (1170) y Bolonia (1088) (Haskins, 2007). La enseñanza que predominó era esencialmente escolástica, cuyo origen se remonta a las escuelas monásticas cristianas. Los maestros leían textos de las autoridades (grandes pensadores), con el fin de transmitir al estudiante el conjunto de saberes de la disciplina y, posteriormente, se realizaban debates (disputatio) en torno a una cuestión (quaestio) (Durkheim, 1995). Como guardián de los valores de su época, la universidad contribuyó al mantenimiento del status quo, reforzando las estructuras de poder, alimentando las filas de los cuerpos eclesiásticos y estatales, o apenas instruyendo a las élites (Giordani, 1982). No había la idea de un "papel de la universidad ante la sociedad, de servicios a ser prestados a la comunidad extramuros" (Castanho, 2000, p. 24).

La institucionalización de la investigación, como una función de la universidad ocurrió en Alemania, en la primera década del siglo XIX (1809/1810), con la creación de la Universidad de Berlín. Su principal idealizador, Wilhelm von Humboldt, entendía que la investigación contribuiría no sólo al avance de la ciencia, sino también a la mejora de la misión de enseñanza, es decir, una retroalimentaria a otra. Según Ribeiro (1982), al implantarse precozmente en la universidad, la ciencia preparó el camino para la industrialización de Alemania al formar recursos humanos especializados para sostenerla, lo que garantizó el desarrollo de una industria química y metalúrgica capaz de competir con las potencias industriales de la época.

Se afirma que la tercera misión surgió en el siglo XIX, en Inglaterra, en Oxford y en Cambridge, cuando comenzaron a delinear las bases de la extensión universitaria. Mackinder y Sadler (1891) afirman que el uso del término "extensión universitaria" surgió en la Universidad de Oxford alrededor de 1845 y se hizo corriente en los años subsiguientes. Entre los años 1867 y 1873, en la Universidad de Cambridge, se aprobaron diversas propuestas de organización de cursos y conferencias para ser llevados a institutos de mecánica, cooperativas, escuelas y otras agremiaciones, especialmente en el norte de Inglaterra, creando las bases para las futuras escuelas o departamentos de extensión universitaria y de educación de adultos en todo el mundo (Welch, 1973).

La extensión universitaria, en la modalidad de prestación de servicios, surgió en los Estados Unidos, con los Land-Grant Colleges, con autorización de una ley federal (Morrill Act - 1862) permitió la donación de tierras federales para el financiamiento de universidades públicas (por regla general, aunque las universidades privadas también han sido financiadas), especialmente para la promoción del desarrollo agrícola e industrial. Según Kerr (2005, p. 25), “el movimiento de concesión de tierras abrió las puertas de las universidades a los hijos de los agricultores y trabajadores, que se sumaron a los hijos de las clases media y alta; introdujo estaciones de experimentación agrícola y oficinas de servicios". Finalmente, fue en los Estados Unidos, a mediados del siglo XX que la universidad pasa a involucrarse con el emprendedorismo, innovación y actividades de transferencia de tecnología, parques tecnológicos, entre otros, teniendo como paradigmas el Massachusetts Institute of Technology (MIT) y la Stanford University.

Actualmente, crecen los debates en favor de la Glocal University, que es la universidad con alcance global y localmente involucrado (Grau, 2014), es decir, no son sólo centros de prestigio internacional pero también están involucrados con las demandas de sus regiones, pues en el siglo XXI se espera que los centros de conocimiento desarrollen una misión regional (Arbo \& Benneworth, 2007). La misión regional puede entenderse como "una tarea formal o un deber atribuido a una colectividad o grupo de personas (en este caso, una universidad), concerniente al desarrollo más amplio de sus alrededores geográficos inmediatos" (Pinheiro, 2011, p. 9).

\subsection{Tercera misión: recorte conceptual}

Etzkowitz Y Leydesdorff (1997) son comúnmente señalados como los creadores del término tercera misión, o al menos como los autores que popularizaron el uso del término, que acabó extendiéndose por los medios académicos y empresariales, pasando a ser asociando con innovación, emprendimiento y asuntos relacionados. Los autores también son conocidos como los creadores del enfoque de la "Triple Hélice" que se preocupa por los arreglos institucionales modelados para la interacción entre universidad-industria-gobierno, atribuyendo a la universidad un papel central en la sociedad basada en el conocimiento. En ese marco, la tercera misión representaría la contribución al desarrollo económico y social. 
Para Roessler, Duong y Hachmeister (2015), la tercera misión es un "fenómeno global con características locales" cuyos entendimientos varían en cuanto al alcance, convirtiéndose cada vez más en un enfoque multidimensional que comporta dimensiones culturales y sociales, así como políticas y económicas. Göransson, Maharajh y Schmoch (2009, p. 158) llaman la atención sobre lo siguiente: "el debate internacional sobre la tercera misión ha sido ampliamente dominado por el modelo norteamericano, pero hay que averiguar si tal modelo puede ser transportado a otros países, especialmente para en desarrollo". Por lo tanto, es necesario considerar que la discusión sobre las actividades que deben o no incluir en la definición de la tercera misión varían considerablemente entre los países y en diferentes contextos.

Fernández-Larrea y González (2013, p. 8) entienden que la interacción universidad-sociedad se realiza a través de la extensión universitaria, que "es el proceso que tiene como propósito promover cultura en la comunidad intra y extrauniversitaria como parte de la contribución universitaria al desarrollo cultural". Los autores sostienen que el modelo de extensión autual está defesado:

no es suficiente ofrecer servicios a determinados sectores externos, no basta a la universidad extender puentes a la sociedad, sin reflexionar sobre los significados de esas acciones, sino que es necesario repensar por entero su actuación con una perspectiva social. Esto exige que la universidad se reconstruya internamente, teniendo en consideración la realidad sociocultural de la cual participa. Por eso no debe solo mirar hacia fuera; debe repensarse desde su interior, lo más importante es reflexionar sobre sus significados y sobre su papel en la construcción de la sociedad en estos nuevos contextos, ya que debe contribuir al conocimiento y a la solución, en su ámbito y de acuerdo a sus posibilidades, de los problemas y necesidades de la sociedad (Fernández-Larrea \& González, 2013, p. 9).

Otro aspecto a tener en cuenta es que, en América Latina, en general, la extensión tiene una connotación diferente de los enfoques de la tercera misión de los países desarrollados, o sea, en nuestra región, la extensión ha sido entendida como difusión cultural y como servicios sociales dirigidos a grupos desfavorecidos. Sin embargo, aunque algunos sectores de la universidad son contrarios a la aproximación de la universidad de las demandas del sector productivo, actualmente, el concepto de extensión se conecta cada vez más al de tercera misión. Por lo tanto, los dos conceptos deben orientar la transferencia de conocimiento a la sociedad, incluyendo entre sus prácticas / acciones / actividades, inclusive, el espíritu emprendedor y la innovación (Grao, Iriarte, Ochoa \& Vieira, 2014).

Schoen y Thèves (2006) y Inzelt et al. (2006) afirman que es por medio de la tercera misión que se determina la forma en que los recursos y las capacidades de la universidad se integran a la economía y a la sociedad. Esta misión es multifacética y presenta dimensiones económicas - como la transferencia de conocimiento y tecnologías, a través de licencias y contratos con la industria; y dimensiones sociales - como la participación en la vida social y cultural. Además, también es importante considerar los diferentes usos del conocimiento científico según aclaran Castro-Martínez, Olmos-Peñuel y Fernandez-De-Lucio (2016, p. 123): en principio, las políticas de fomento de las relaciones cienciasociedad se diseñan con objeto de favorecer las interacciones de los investigadores con las industrias en sus procesos innovadores, por lo que implícitamente están considerando sólo el uso instrumental del conocimiento científico, pero cuando se amplía el tipo de sectores para dar cabida a los servicios - incluyendo a las administraciones públicas y otros agentes sociales - se observa que los usos conceptual y simbólico pueden presentar mayor relevancia que el instrumental.

En el Proyecto titulado "European Indicators and Ranking Methodology for University Third Mission - E3M", patrocinado por la Comisión Europea, se afirmó que la tercera misión se realiza cuando las actividades de producción, utilización, aplicación y explotación de conocimientos y otras capacidades de la universidad alcanzan el público externo a la academia. Además, se estableció que la tercera misión está constituida por tres dimensiones: (i) educación continuada; (ii) transferencia de tecnología e innovación; (iii) compromiso social (European Indicators and Ranking Methodology for University Third - E3M, 2012a, b, c). El Proyecto VINCULAENTORNO, orientado a la cooperación entre la Unión Europea y América Latina para la promoción de la educación superior como un instrumento de desarrollo social y económico, adoptó la clasificación establecida por el European Indicators and Ranking Methodology for University Third Mission - E3M (2012a, b, c) y también se consideró que la tercera misión presenta tres dimensiones (educación continuada, transferencia de tecnología e innovación, compromiso social) (Mora y Vieira, 2014).

En el Brasil, en virtud de la Constitución Federal de 1988, las universidades deben realizar la enseñanza, la investigación y la extensión y se percibe que ese direccionamiento legal acabó por establecer que la extensión universitaria es la tercera misión de la universidad. Sin embargo, el concepto de extensión universitaria, así como su alcance todavía carecen de entendimiento. Entendemos que la utilización del término extensión se ha mostrado limitada para representar todas las posibles relaciones de la universidad con la sociedad, en función de la enseñanza, de la investigación, y de sus capacidades físicas y de conocimiento.

En el ámbito de las universidades públicas brasileñas, el Foro Nacional de Pro-Rectores de Extensión de las Universidades Públicas (FORPROEX), formado por Pró-rectores/Vicerrectores de Extensión de las universidades participantes, por ejemplo, ha sido el responsable por la creación de un cuadro conceptual, así como por la organización y sistematización de la extensión. Según el Foro, "la extensión universitaria es el proceso educativo, cultural y científico que articula la enseñanza y la investigación de forma indisociable y viabiliza la relación transformadora entre la universidad y la sociedade" (Forproex, 1987, p. 11). Aunque el Foro reconoce que las actividades relacionadas con el espíritu emprendedor, la innovación, la propiedad intelectual y la transferencia de tecnología en las universidades pueden considerarse como extensión, no siempre se considerarán tales actividades, ya que los resultados de estas actividades se deben originar en el marco de programas o de proyectos de extensión, situación relativamente rara de ocurrir, especialmente porque las investigaciones que pueden 
generar invenciones, patentes y transferencia de tecnología involucra sigilo, mientras que el propósito de la extensión es la publicidad, la coproducción y el compartir. Por lo tanto, cuando tales actividades (emprendedorismo, innovación, patentamiento, etc.) no se realizan en el ámbito de la extensión, ¿cuál sería su clasificación o el nombre que esas relaciones deberían recibir? Otra cuestión que también merece destacarse es que incluso las actividades más tradicionales y típicamente integrantes de la extensión, como los cursos, no son reconocidas y aceptadas de forma unánime por algunos grupos dentro de las universidades, especialmente las disciplinas y los cursos pagados, habiendo preferencia por las acciones culturales, artísticas y comunitarias, en algunos casos.

En vista de estas evidencias es que optamos por nombrar la relación universidad-sociedad de "tercera misión", tomando como ejemplo iniciativas internacionales en ese sentido (Proyecto E3M y Proyecto VINCULAENTORNO, citados anteriormente) que se preocuparon por crear un marco conceptual que permitiese una visión holística de la relación universidad-sociedad. Siendo así, entendemos que la tercera misión es la interfaz que une a la universidad de forma más directa a la sociedad, con el apoyo de las instalaciones físicas y las capacidades de conocimiento, o sea, representa la articulación de las misiones enseñanza e investigación y de diversos mecanismos e instrumentos que comprenden tanto la estructura física - bibliotecas, museos, laboratorios, instalaciones destinadas al deporte, a la cultura y al ocio, entre otras; como también por intermedio de las existencias de conocimiento y de las capacidades y habilidades de la comunidad académica.

\section{Procedimientos Metodológicos}

\subsection{Identificación de las actividades de la tercera misión: criterios utilizados}

Para el escrutinio y clasificación de actividades de la tercera misión en la Universidade Estadual de Campinas (Unicamp) se adoptaron las tres dimensiones conceptuales establecidas por el "European Indicators and Ranking Methodology for University Third Mission - E3M", que se llevó a cabo entre enero de 2009 y diciembre de 2012. Este proyecto estableció que la tercera misión está formada por tres dimensiones, que son: (i) dimensión transferencia de tecnología e innovación; (ii) dimensión de la educación continuada; (iii) dimensión compromiso social. Posteriormente, entre 2012 y 2014, el Proyecto VINCULAENTORNO, que contó con la participación de universidades de América Latina (incluida la Unicamp), también adoptó la referida clasificación. Los dos proyectos citados (E3M y VINCULAENTORNO) representan acciones en pro de la comprensión de la tercera misión desde un punto de vista holístico de las diversas posibilidades de acción, por lo que adoptamos la referida clasificación.

La dimensión innovación y transferencia de tecnología promueve la diseminación y aplicación del conocimiento, experiencias, entre otras, resultantes de las actividades de investigación desarrolladas en la universidad. Es la interfaz entre la investigación universitaria y el mundo no académico (comunidad, sociedad) y eso puede involucrar: protección a la propiedad intelectual; contratos de licencias; establecimiento de estructuras de apoyo a la innovación y al espíritu empresarial (incubadoras, parques científicos y tecnológicos, oficinas de transferencia de tecnología); compartiendo y alquilando laboratorios y otros espacios, entre otras acciones (E3M, 2012a, b, c). En la dimensión educación continuada interesan los procesos formativos que posibiliten el perfeccionamiento de habilidades y competencias individuales y el aumento del nivel de conocimiento, es decir, se refiere a la educación y la formación. En esta dimensión importa esencialmente determinar cómo y cuáles son las iniciativas desarrolladas por las universidades para extender los beneficios del conocimiento y de la educación superior a grupos no tradicionales de estudiantes, es decir, a los que no son alumnos regulares de la graduación o del postgrado. La dimensión compromiso social se refiere a la utilización y asignación de los recursos del conocimiento (intelectuales y físicos) de la universidad para contribuir con la comunidad o región, y en términos más amplios, con la sociedad en general. En esta dimensión es bastante común que se fusionen las actividades e iniciativas de las otras dos dimensiones, pues las fronteras entre las dimensiones no son limitadas, lo que hace que esta dimensión asuma una naturaleza híbrida. El compromiso social puede ocurrir cuando la universidad se involucra con demandas o necesidades de empresas, gobiernos locales, por ejemplo; cuando permite a la comunidad el acceso a bibliotecas, museos, teatros y otras instalaciones; al participar y estimular debates relacionados con cuestiones sociales críticas (medioambientales, por ejemplo); cuando promove el voluntariado. El ofrecimiento de cursos y disciplinas de extensión gratuitas también puede ser encuadrado en esta dimensión, así como las acciones dirigidas a la divulgación y popularización de la ciencia, la participación con acciones de economía solidaria, generación y difusión de tecnologías sociales, atención a cuestiones de salud pública, ambientales y otras necesidades urgentes de la comunidad y del entorno de la universidad también son ejemplos de compromiso social.

\subsection{Selección de los entrevistados}

Para la selección de entrevistados, se identificaron, primero, las principales instancias que tratan con la tercera misión y sus respectivos representantes: Directores, Pro-Rectores, asesores u otros profesionales involucrados de alguna forma con el tema. La Tabla 1 presenta la lista de instancias y la función de los entrevistados que aceptaron participar en la investigación.

Tabla 1. Unicamp: instancias seleccionadas

\begin{tabular}{l|l}
\hline Unicamp & Función/Posición \\
\hline \multirow{2}{*}{ Escuela de Extensión de la Unicamp } & Director \\
\cline { 2 - 2 } & Director \\
\hline Inova Unicamp & Gerente de comunicación \\
\hline Instituto de Economía & Profesor $^{1}$ \\
\hline Laboratorio de Periodismo Científico & Investigador y profesor \\
\hline Pro-Rectoría de Extensión y de Asuntos & Asesor del Pro-rector \\
\cline { 2 - 2 } Comunitarios & Secretaría \\
\hline Fundación de Desarrollo de la Unicamp & Director ejecutivo \\
\hline Coordinación General de la Unicamp & Vicerrector \\
\hline
\end{tabular}

Nota 1: representó a la Unicamp en el Proyecto VINCULAENTORNO, que investigó la realización de la tercera misión en universidades latinoamericanas y europeas. 
Se realizaron 9 entrevistas entre los meses de diciembre de 2015 y octubre de 2016 y el cuestionario aplicado a los entrevistados estaba compuesto por 17 cuestiones abiertas que pretendían identificar la opinión del encuestado acerca de prácticas específicas de la universidad, así como sobre las estructuras e instancias que se conectan con la sociedad.

A continuación, se presenta una síntesis de los principales resultados obtenidos con la investigación conducida en la Unicamp, iniciándose por la presentación de acciones y prácticas seleccionadas de la tercera misión (letra a), en la secuencia, se presentan las percepciones y visiones de los entrevistados letrab).

\section{Resultados y Discusión}

\subsection{Universidade Estadual de Campinas (Unicamp): breve histórico y perfil}

La Unicamp y una universidad pública del Estado de São Paulo, Brasil, creada em 1966. La universidad tiene aproximadamente 37 mil alumnos matriculados en 66 cursos de graduación y 152 programas de postgrado, mientras que el $47 \%$ de todos los alumnos están concentrados en posgrado. La Unicamp posee 1.910 profesores, de los cuales 99 \% como mínimo con titulación de doctor (94\% régimen actuando en Dedicación exclusiva) posee 24 unidades de enseñanza e investigación, 21 centros y núcleos interdisciplinarios, 3 hospitales, 2 escuelas técnicas y 29 bibliotecas (Unicamp, 2017). La Unicamp es una universidad joven (cumplió 50 años en 2016), pero ya conquistó tradición en la enseñanza, en la investigación y en las relaciones con la sociedad, pues es responsable de aproximadamente $8 \%$ de toda la producción científica del Brasil y de 12\% de la graduación nacional. También, su complejo de salud atiende a cerca de 500 mil pacientes al año. El desempeño de la institución también viene repercutiendo internacionalmente, pues integra un grupo bastante reducido de universidades brasileñas que han aparecido en los rankings internacionales (como el Quacquarelli Symonds - QS y el Times Higher Education - THE): en el THE (2017), por ejemplo, figuró en la $1^{\text {a }}$ colocación en el ranking de América Latina; en el ranking QS (2018) de las mejores universidades de América Latina y de las 100 mejores de los BRICS, la Unicamp apareció, respectivamente, en la $2^{\mathrm{a}}$ y en la $12^{\mathrm{a}}$ colocación.

4.2 Las relaciones con la sociedad en la práctica: estructuras, instancias y dimensiones de la tercera misión en la Unicamp

En este ítem presentaremos instancias y acciones seleccionadas y no el conjunto de iniciativas, ni el conjunto de instancias y estructuras de la Unicamp a las que fueron institucionalmente atribuidas la función de conducir la tercera misión, pues eso extrapolaría los objetivos de la investigación. Además, es importante considerar que existen diversas acciones conducidas por instancias que tienen otras funciones institucionales que no específicamente la de servir de interfaz entre la universidad y la sociedad. Además, las unidades de enseñanza e investigación, en su gran mayoría, desarrollan periódicamente acciones dirigidas a la sociedad, tales como: eventos públicos y gratuitos, cursos, talleres, conferencias, seminarios, olimpiadas, entre muchos otros.

Sin embargo, existen instancias específicas cuya misión primordial es realizar la relación universidad-sociedad en la Unicamp, como la ProRectoría de Extensión y Asuntos Comunitarios (PREAC), que fue creada en 1986 para "coordinar, fomentar, estimular y producir acciones de Extensión y de Cultura por la integración dialógica, interactiva y proactiva con la sociedad, difundiendo y adquiriendo conocimiento a través de la comunidad universitaria" (http://www.50anos.unicamp.br/ linha-de-tempo). La PREAC tiene bajo su dirección varios órganos y estructuras, tales como: el Cultural de Inclusión e Integración Social Cis - Guanabara; el Espacio Cultural Casa del Lago - Ecult; la Escuela de Extensión (Extecamp); el Laboratorio de Estudios e Investigaciones en Artes y Ciencias - Lepac; las emisoras de radio y televisión de la Unicamp, entre otras. Por intermedio de estos diversos órganos se realizan diversas acciones y actividades que vinculan a la Unicamp a la sociedad, sea en el campo de la divulgación y comunicación (vía radio y televisión), sea por medio del ofrecimiento de cursos de extensión universitaria, o por medio de acciones comunitarias, artísticas y culturales, entre otros. Otra instancia orientada a las relaciones con la sociedad es la Agencia de Innovación de la Unicamp (Inova Unicamp), creada en 2003, con la misión de "identificar oportunidades y promover actividades de estímulo a la innovación y al espíritu emprendedor, ampliando el impacto de la enseñanza, de la investigación y de la extensión en favor del desarrollo socioeconómico sostenido". La agencia está vinculada al Gabinete del Rector, siendo responsable por la gestión de la política de propiedad intelectual (PI) de la Unicamp, así como por la administración de la Incubadora de Empresas de la Unicamp (Incamp) y del Parque Científico y Tecnológico, además de promover la cultura en la universidad (ofreciendo cursos, capacitaciones, premiando inventores, promoviendo concursos y competiciones, entre otras acciones).

Finalmente, otra área extremadamente importante es la de la salud, cuyo alcance de los servicios prestados por la Unicamp a la comunidad de la Región de Campinas (en el estado de São Paulo) es indiscutible. Además del alcance social del área de la salud, la prestación de estos servicios se convierte también en una valiosa oportunidad para que los estudiantes adquieran competencias específicas y perfeccionen habilidades indispensables para el desempeño de la profesión. A continuación, presenta una selección de acciones de la tercera misión en la Unicamp y su encuadramiento en las respectivas dimensiones, a saber: innovación y transferencia de tecnología, educación continuada y compromiso social.

\subsubsection{Dimensión innovación y transferencia de tecnologia}

Con el apoyo de la Agencia de Innovación (Inova) la Unicamp se ha destacado en el ambiente de innovación nacional como una de las universidades brasileñas más exitosas en la conducción de la política institucional de protección y gestión de la protección de la propiedad intelectual generada. La experiencia de la Unicamp en ese campo se comprueba por medio de diversos indicadores, especialmente los del Instituto Nacional de la Propiedad Industrial (INPI), que la colocan entre los principales depositantes de solicitudes de patentes del país, ocupando las primeras colocaciones entre las instituciones nacionales que más depositan solicitudes de patentes ante el INPI. Entre 2000 y 2012 la Unicamp fue la primera entre las universidades y la tercera en la clasificación general (Carvalho, Jorge, Barcelos, Lopes \& Pinheiro, 2015); en 2015 ocupó la tercera colocación entre las mayores patentes de Brasil y la segunda entre las universidades. En 2017, la Unicamp, fue nuevamente la primera colocada (Instituto Nacional de la Propiedad Industrial, 2018). La Figura 1 ilustra el flujo de actividades de Inova Unicamp, específicamente, su actuación en la protección de las invenciones generadas en la universidad. 
Figura 1. Protección a las invenciones generadas en la Unicamp: passos

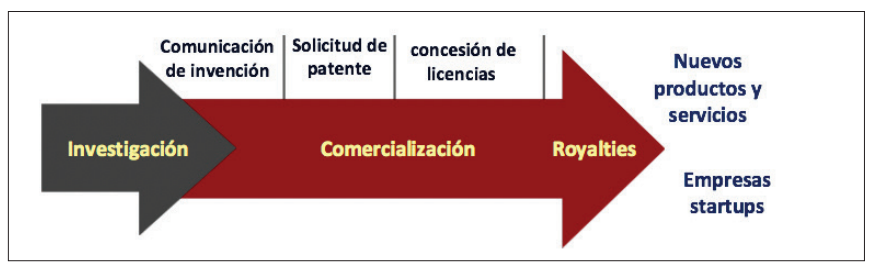

Fuente: Adaptado y traducido de Inova Unicamp (2018).
La Tabla 2 trae algunos resultados referentes a la conducción de la política de propiedad intelectual de la Unicamp, del período comprendido entre 2012 y 2016.

Tabla 2. Resultados obtenidos por Innovación en el período 2012-2016

\begin{tabular}{|c|c|c|c|c|c|}
\hline Actividades & 2012 & 2013 & 2014 & 2015 & 2016 \\
\hline $\begin{array}{l}\text { Solicitudes de patentes depositadas en el Instituto Nacional de la Propiedad } \\
\text { Industrial (INPI) }\end{array}$ & 74 & 71 & 79 & 58 & 80 \\
\hline Solicitudes de patentes depositadas a través Patent Cooperation Treaty (PCT) & 22 & 16 & 12 & 21 & 31 \\
\hline Licencias de tecnología: contratos vigentes & 52 & 59 & 57 & 71 & 87 \\
\hline Comunicaciones de invención recibidas & 107 & 122 & 104 & 80 & 144 \\
\hline Solicitudes de registro de programa informático & 29 & 16 & 20 & 12 & 21 \\
\hline Empresas incubadas & 10 & 8 & 9 & 15 & 13 \\
\hline Empresas graduadas (acumulado) & 36 & 37 & 40 & 44 & 44 \\
\hline
\end{tabular}

Fuente: elaborado por las autoras con base en el Informe de Actividades de la Inova Unicamp 2016 (2017).

Se puede observar, por ejemplo, que la Unicamp contaba con 1.042 patentes vigentes, en 2016, así como con 87 contratos de licenciamiento de tecnología y hubo 144 comunicaciones de invención. En ese mismo año, el número total de depósitos de solicitudes de patentes fue 118 (80 junto al INPI, 7 en el exterior y 31 por intermedio del PCT). Además de los números expresivos en el campo de la protección y gestión de la propiedad intelectual, la Unicamp también ha contribuido al establecimiento de un ambiente favorable al emprendedorismo, a la innovación y a la transferencia de tecnología al instituir y diseñar eventos y estructuras formales para la realización de tales objetivos. En el campo del emprendedorismo, por ejemplo, la Unicamp desempeña un importante papel en la generación de startups (o "empresas hijas"). En 2016 existían 434 "empresas hijas" de la Unicamp activas en el mercado. Estas empresas generaron aproximadamente 22 mil empleos directos y tuvieron una facturación de más de US\$ 700 millones. Se consideran empresas-hijas las empresas cuyos socios (fundador o actual) y/o las actividades están relacionados con la Unicamp de la siguiente forma: empresas de alumnos o ex alumnos (graduación o posgrado); empresas de profesores o ex profesores; empresas de personal o ex funcionarios; empresas incubadas o graduadas en la Incubadora de la Unicamp (Incamp); emprendedores que firmaron contrato de licenciamiento de tecnologías de la
Unicamp, siempre que estas tecnologías sean parte fundamental de la empresa. El registro de las empresas-hijas es realizado por Inova Unicamp.

La Inova Unicamp también conduce varias otras iniciativas, como el Premio Inventores Unicamp que homenajea a profesores, investigadores, ex alumnos y alumnos que son premiados en cuatro categorías: tecnología licenciada, patentes concedidas, tecnología absorbida por el mercado y destaque en la protección a la propiedad intelectual (para las unidades de enseñanza e investigación con mayor participación en la cultura de protección de los resultados de sus investigaciones). Otra importante premiación es el "Desafío Unicamp de Innovación Tecnológica”, una competencia de alcance nacional que dura en promedio tres meses y que pretende motivar a estudiantes, vinculados o no a la Unicamp, a crear modelos de negocio de base tecnológica a partir de patentes o programas de ordenador pertenecientes al Porfolio de la universidad. Durante la competición ocurren diversas actividades como talleres, charlas y mentorías. Al final del período es elegido el equipo, el que consigue conquistar el primer lugar recibe un premio en efectivo, que en 2017 fue de $\mathrm{R} \$ 3.000,00$ para cada integrante del equipo, además de certificado, trofeo entre otros. Es importante subrayar que las iniciativas anteriormente citadas no 
representan la totalidad de las acciones llevadas a cabo por Inova Unicamp, pues la agencia actúa activamente dentro de sus atribuciones, ejerciendo un importante papel en la conducción de la política de propiedad intelectual de la Unicamp, realizando el puente entre la universidad y el sector productivo, bien divulgando y desarrollando acciones relacionadas con la innovación y el emprendimiento. Las acciones de la Agencia de Innovación de la Unicamp encuentran su razón de ser en la intensa actividad de investigación realizada en la universidad.

\subsubsection{Dimensión de la educación continuada}

Para representar esta dimensión se optó por averiguar la extensión universitaria, especialmente, los cursos o disciplinas ofrecidas por la Escuela de Extensión de la Unicamp (Extecamp). Los cursos y disciplinas de extensión representan formas más flexibles y rápidas de adquisición de nuevos conocimientos, actualización de conceptos y prácticas, así como calificación profesional. Para las universidades, por otro lado, pueden representar nuevos canales de ingreso de recursos financieros, además de exteriorizar su responsabilidad social o compromiso público hacia la expansión de los beneficios del conocimiento a la sociedad, fomentando las capacidades de conocimiento y de mano de obra calificada en la región, así como proporcionando condiciones de empleabilidad. En las universidades brasileñas la mayor parte de las acciones de esta dimensión quedan bajo la responsabilidad de las Pro-Rectorías de Extensión (o Vicerrectorías), siendo que los cursos y disciplinas son comúnmente centralizados y ofrecidos por las Escuelas de Extensión. La Escuela de Extensión de la Unicamp (Extecamp), creada en 1989 y está vinculada a la Rectoría de Extensión y Asuntos Comunitarios (PREAC), siendo la responsable de la administración y el ofrecimiento de los cursos formales de extensión. La Tabla 3 presenta las modalidades y los requisitos de los cursos de extensión ofrecidos por Extecamp.

Tabla 3. Modalidades de cursos de extensión ofrecidos por Extecamp

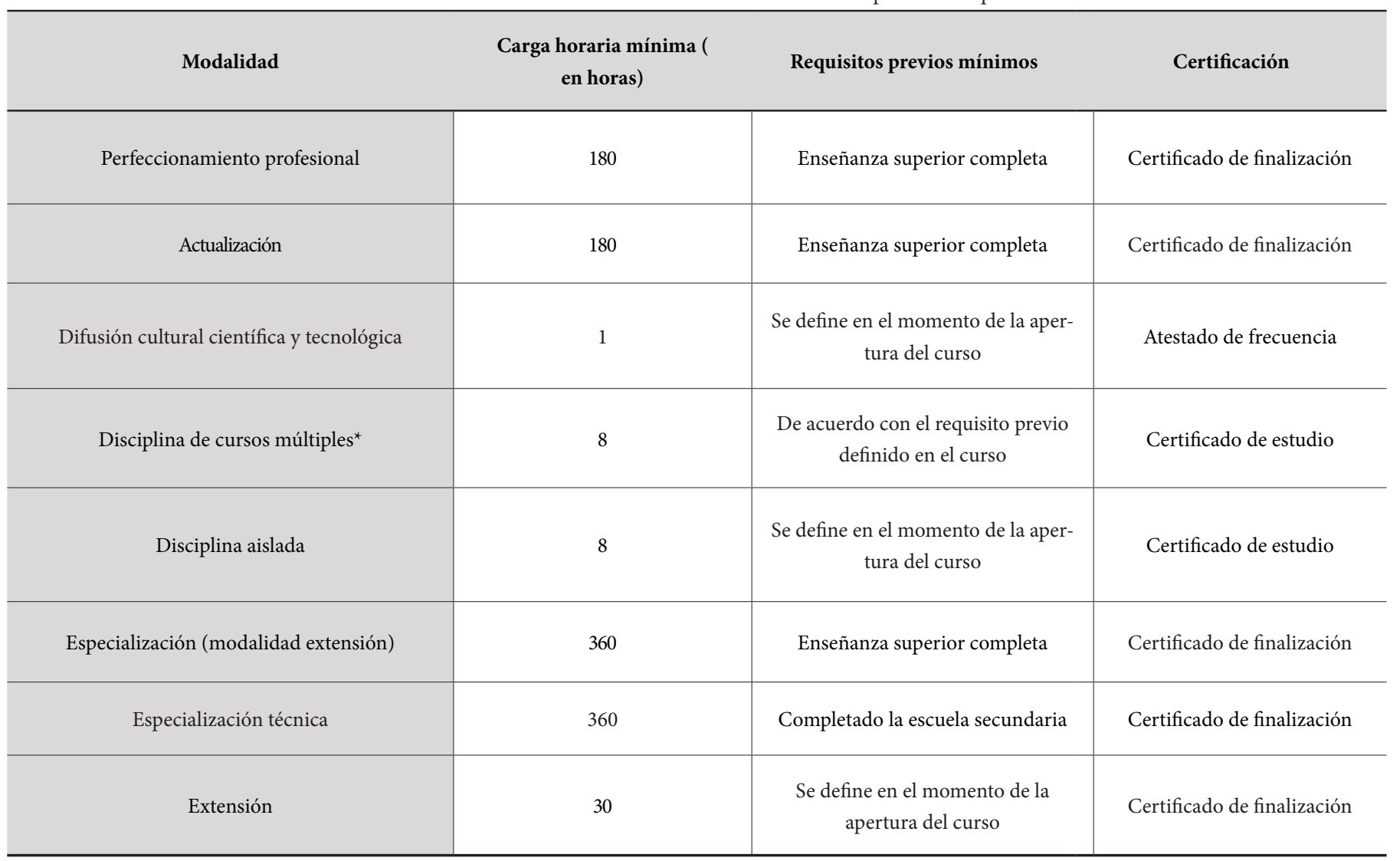

Nota * el curso es simple cuando el curso y la disciplina forman una sola unidad; o múltiple, cuando está compuesto por dos disciplinas o más, pero es necesario que cada asignatura tenga carga horaria de por lo menos 8 horas. Fuente: adaptado del Informe de Actividades 2016 de Extecamp (2017, p. 11-12).

En 2016, Extecamp inició 353 cursos y asignaturas distribuidas de la siguiente forma: 11 cursos de perfeccionamiento; 80 cursos de especialización; 165 cursos de extensión; 82 cursos de difusión y 15 disciplinas aisladas, resultando en 29.875 matrículas (que fueron efectuadas por 10.038 alumnos). El número de matrículas realizadas en 2016 fue de 34.315 y el de estudiantes fue de 11.356, siendo que cada estudiante realizó, en promedio, los cursos que se iniciaron en el período anterior, el número de matrículas realizadas en 2016 fue de 34.315 y el de estudiantes fue de 11.356, 32 matrículas, a lo largo del año. El área con el mayor número de cursos y disciplinas propuestos en 2016 fue la de Ciencias Tecnológicas, representando el 31,9\% del total (733), siendo que el número de cursos y disciplinas efectivos fue 448 , o sea, $61,1 \%$ del total, total propuesto en el período. Esta área también presentó el mayor número de matrículas en el mismo 
período totalizando 12,861, lo que representó el 37,5\% del total. Otra área que se destacó en 2016 fue la de Ciencias Biomédicas, que respondió por el 73\% del total de horas-lecciones impartidas, o sea, 91.447 horas-clase (de 125,312 horas-clase ministradas). El área con la mayor tasa de éxito en el ofrecimiento de cursos / disciplinas fue la de Ciencias Aplicadas, que propuso 138 cursos y disciplinas, de los cuales 114 se efectuaron (82,6\%). Finalmente, con el objetivo de promover la ampliación del número de beneficiados con los conocimientos y experiencias de la Unicamp, también comúnmente se ofrecen cursos y disciplinas gratuitos. Para el cálculo de los números y porcentajes relativos a los cursos y disciplinas gratuitos se consideraron todas las modalidades de cursos, excepto las disciplinas de cursos múltiples. Por lo tanto, en 2016 se ofrecieron 84 cursos/ disciplinas gratuitas, lo que representó el 23,8\% del total de cursos efectuados (533) por la Escuela de Extensión de la Unicamp en ese período. Estos cursos fueron frecuentados por 2,041 estudiantes, representando el $21,7 \%$ del total de estudiantes del período $(9,412)$ (Extecamp, 2017b).

\subsubsection{Dimensión compromiso social: servicios de salud}

En vista de la amplitud de esta dimensión, que imposibilitaría la realización de una investigación minuciosa de las diversas actividades y prácticas desarrolladas en muchas de las 24 unidades de enseñanza e investigación de la Unicamp, así como todas las acciones de la ProRectoría de Extensión y Asuntos Comunitarios (PREAC) de otras instancias de la universidad, optamos por la presentación de algunos indicadores del área de salud, especialmente los que involucra los servicios prestados a la comunidad.

El complejo de salud de la Unicamp está inserto en la Facultad de Ciencias Médicas (FCM) y está formado por los siguientes órganos: Hospital de Clínicas (HC); Hospital Estadual Sumaré (HES); Hospital de la Mujer "Profesor Doctor José Aristodemo Pinotti" - Centro de Atención Integral a la Salud de la Mujer (CAISM); y los servicios de apoyo (Hemocentro, Centro de Diagnóstico de Enfermedades del Aparato Digestivo - Gastrocentro, Centro de Estudios e Investigaciones en Rehabilitación "Prof. Dr. Gabriel O.S. Puerto" (CEPRE). El Hospital de Clínicas (HC) de la Unicamp es considerado uno de los más importantes hospitales universitarios del país, siendo que la actividad de enseñanza es una de las principales finalidades del hospital, pues los diversos procedimientos quirúrgicos, exámenes y las investigaciones realizadas proporcionan entrenamiento y etapa para alumnos de graduación, residentes y postgraduados en múltiples áreas de las Ciencias de la Salud y de otras profesiones relacionadas. En el HC se entrenan anualmente alrededor de 670 estudiantes de Medicina, 161 de Enfermería, 83 de Fonoaudiología y 40 de Farmacia. El HC también poseía el tercer mayor número de médicos residentes de Brasil (607), además de contar con aproximadamente 800 post graduados. El HC atiende a más de 100 municipios (lo que corresponde aproximadamente a 5 millones de personas), con una circulación diaria de más de 10 mil personas (https://www.hc.unicamp.br/ node/178). La Tabla 4 presenta las cifras referentes a las actividades del complejo de salud de la Unicamp en 2016.
Tabla 4. Servicios de Salud realizados en 2016

\begin{tabular}{l|c}
\hline Tipo de Servicio & Números \\
\hline Lechos - hospitales & 863 \\
\hline Trasplantes: & 351 \\
Cardíaco & 123 \\
Córnea & 47 \\
Hígado & 37 \\
Medula ósea & 136 \\
Riñón & 38.166 \\
\hline Internaciones hospitalarias & 950.526 \\
\hline Consultas atendidas & 59.643 \\
\hline Intervenciones quirúrgicas & 5.129 \\
\hline Nascimientos & 355.098 \\
\hline Servicios de imagen & 6.344 .551 \\
\hline Exámenes de laboratorio & 148.110 \\
\hline Procedimientos odontológicos & \\
\hline
\end{tabular}

Nota: incluye radiografía, ultrasonografía, resonancia magnética, radiología, ecografía y mamografía. Fuente: Anuario Estadístico 2016 (2017).

El Hospital de Clínicas (HC) es una referencia nacional en la formación y especialización de recursos humanos y también desempeña un papel destacado en el desarrollo de investigación y tecnología a través de investigaciones a nivel de iniciación científica, maestría, doctorado y postdoctorado, elaboración y desarrollo pruebas de nuevos tratamientos, medicamentos, prácticas quirúrgicas entre otros. De esta forma, "la efectiva prestación de servicios de asistencia a la población posibilita el constante perfeccionamiento de la atención, con la formulación de protocolos técnicos para las diversas patologías, lo que garantiza mejores estándares de eficiencia y eficacia, puestos a disposición para la Red del Sistema Único de Salud (SUS)" (https://www. hc.unicamp.br/node/71).

Los números y las informaciones presentadas permiten percibir el importante alcance de los servicios de salud prestados por la Unicamp a la comunidad. Sin embargo, como se observa, la prestación de estos servicios se convierte también en una valiosa oportunidad para que los estudiantes adquieran competencias específicas y mejoren las habilidades indispensables para el ejercicio de la profesión, constituyéndose en una vía de doble vía en la que la relación con la sociedad es una importante fuente de retroalimentación de las misiones enseñanza e investigación.

\subsubsection{Percepciones y visiones de los entrevistados acerca de la relación universidad-sociedad: la realización de la tercera misión en la Unicamp}

Como se describe en la sección de los procedimientos metodológicos, las 9 entrevistas se realizaron entre los meses de diciembre de 2015 y octubre de 2016 a partir de un itinerario compuesto por 17 preguntas abiertas, posibilitando al entrevistado flexibilidad y libertad para responder y explicar las razones de sus respuestas. En el caso de que se 
trate de una de las más importantes para el logro de los objetivos propuestos para este artículo, es preferible la presentación de una síntesis de las entrevistas y elegimos sólo las 3 preguntas (Q.1, Q.2 y Q.3) de los entrevistados acerca de la realización de la tercera misión en la Unicamp. Es importante aclarar que las demás preguntas, aunque hayan sido relevantes para la investigación original (la tesis de doctorado), algunas de ellas se volvían a aspectos más genéricos o a cuestiones profesionales específicas (relacionados con las funciones de los entrevistados) o a temas más amplios involucrando el contexto nacional e internacional. En vista de ello, entendemos que la presentación en su totalidad extenderá excesivamente el artículo, haciendo la discusión excesivamente larga. Por lo tanto, a continuación, se exponen las preguntas y respuestas:

Q.1. ¿Como usted entiende la estructura de la Unicamp (Pro-Rectoría de Extensión y Asuntos Comunitarios, Escuela de Extensión, Fundación de Desarrollo de la Unicamp, Agencia de Innovación y otras) direccionada a la relación Universidad-Sociedad? ¿Es adecuada? ¿Está muy descentralizada? ¿Hay superposiciones? ¿Que está faltando? ¿Qué sería lo más adecuado?

Según la mayoría de los entrevistados (8), entendió y consideró adecuada la actual estructura universitaria (instancias) para la realización de la relación universidad-sociedad. Sin embargo, incluso considerando la estructura adecuada, se señalaron algunos obstáculos / obstáculos al desarrollo y consolidación de la relación de la universidad con la sociedad (falta de diálogo, comunicación e interacción entre las instancias fueron los más señalados). Sólo 1 entrevistado entendió que la estructura debería ser más centralizada, pues la centralización podría hacer las acciones más cohesionadas, facilitar la comunicación, la alineación de intereses y las interacciones. Por centralización se presume que todas las acciones dirigidas a la sociedad deberían ser colocadas bajo la tutela de la Pro-Rectoría de Extensión, indepentemente del público objetivo (empresas, organizaciones sin fines de lucro, comunidad en general, gobierno, entre otros).

Q.2. ¿Qué cambios sugeriría en la forma en que la Unicamp conduce sus actividades de interacción con la Sociedad? ¿Por qué y cuáles?

Todos los entrevistados realizaron alguna (o muchas) sugerencias, en diversos campos, tales como: crear y/o mejorar los canales de comunicación internos y externos; interconectar las instancias y promover sinergias; profundizar el nivel de conocimiento y perfeccionar las concepciones vigentes acerca de la tercera misión, adoptando una visión holística; reconocimiento y valorización del docente en las actividades de extensión universitaria; crear metodologías de evaluación; crear una cultura de la tercera misión; desarrollar y perfeccionar las formas de gobernanza; la legitimación de la universidad frente a la sociedad (difundir, informar, demostrar la relevancia de su actuación - de las investigaciones que realiza, de la enseñanza, etc.).

Q.3. ¿Cuáles son las principales dificultades para el desarrollo de la relación universidad-sociedad?

Fueron muchas las dificultades (obstáculos, desafíos, como prefirieron llamar algunos entrevistados) apuntados. Algunos de los entrevistados afirmaron que la universidad sigue siendo una "torre de marfil" y se presenta de forma muy arrogante ante la sociedad. La falta de incentivo al docente también fue considerada un obstáculo (los incentivos, la valorización, el reflejo en la carrera docente ocurre, por regla general, a partir de las actividades de enseñanza e investigación - publicaciones); preconcepto respecto de la realización de actividades de protección de las tecnologías generadas y de la transferencia de tecnología; preconcepto respecto de ciertas relaciones, por ejemplo, con el sector productivo; visiones estrechas y limitadas de la extensión universitaria - todavía existen en las universidades (públicas) visiones fuertemente asistencialistas; la sociedad/comunidad/el público en general desconoce todas las realidades de la contribución de la universidad (luego, es necesario si "mostrar", divulgar, difundir lo que se hace); carencia de recursos financieros; visión estrecha de la relación universidad-sociedad (necesidad de una visión más amplia).

\section{Conclusiones}

Los resultados permitieron concluir que la Unicamp se destaca en la conducción de varias iniciativas de la tercera misión, en diversos campos, pasando por la excelencia en el área de salud y de los servicios ofrecidos a la población, al gran número de cursos ofrecidos por la Escuela de Extensión (Extecamp), a las acciones de la Pro-Rectoría de Extensión y Asuntos Comunitarios (PREAC) volcadas a la extensión comunitaria. Además, la actuación de Inova ha posibilitado que la Unicamp desempeñe un papel destacado en el escenario nacional como una de las universidades más activas en el campo de la protección a la propiedad intelectual, licencias, incubación de empresas, gestión del parque tecnológico, entre otras importantes acciones.

Sin embargo, aunque las estructuras e instancias de soporte a tales acciones están bien consolidadas, se nota la falta de interacciones más fuertes entre ellas, no en el sentido de una estructura única y centralizadora para albergar tales acciones, sino en lo que se refiere al establecimiento de directrices que refuercen iniciativas conjuntas y promuevan sinergias. La tercera misión es compleja, multifacética, pues son innumerables las posibilidades de realización, y hay mucho aún por ser pensado. Son muchos los puntos de contacto que pueden ser trabajados, tales como, mejorar los canales de comunicación, fomentar el diálogo e intercambio de informaciones, estimular y desarrollar iniciativas conjuntas entre las diferentes instancias, entre otros.

\section{Referencias}

Arbo, P. \& Benneworth, P. (2007). Understanding the regional contribution of higher education institutions. OECD Education Working Papers, n. 9, p. 1-76. http://dx.doi.org/10.1787/161208155312

Carvalho, S. M. P., Jorge, M. F., Barcelos, V. I., Lopes, F. V. \& Pinheiro, V. L. S. (2015). Indicadores de Propriedade Industrial (2000-2012): o uso do sistema de Propriedade Industrial no Brasil. Rio de Janeiro: Instituto Nacional da Propriedade Industrial - INPI. Recuperado de http://www.inpi.gov.br/sobre/estatisticas/arquivos/publicacoes/indicadores-de-propriedade-industrial-20002012.pdf 
Castanho, S. E. M. (2000). A universidade entre o sim, o não e o talvez. In: Veiga, I. P. A. \& Castanho, M. E. L. M. (Orgs.). Pedagogia universitária: a aula em foco (pp. 13-48). Campinas, SP: Papirus.

Castro-Martínez, E., Olmos-Peñuela, J. \& Fernandez-De-Lucio, I. La Vinculación Ciencia-Sociedad: Estereotipos y Nuevos Enfoques. Journal of Technology Management \& Innovation, 11, n. 2, p. 121-129, 2016. https://dx.doi.org/10.4067/S0718-27242016000200012

D’este, P. Martínez, E. C. \& Molas-Gallart, J. (2014). Documento de base para un Manual de indicadores de vinculación de la universidad con el entorno socioeconómico. (Manual de Valencia). Espanhã: INGENIO (CSIC-UPV). Recuperado de http://digital.csic.es/handle/10261/132865

Durkheim, E. A. (1995). Evolução Pedagógica. Tradução de Bruno Charles Magne. Porto alegre: Artes Médicas.

Etzkowitz, H. \& Leydesdorff, L. (1997). Introduction: Universities in the Global Knowledge Economy. In: Etzkowitz \& H.; Leydesdorff (orgs). Universities in the Global Knowledge Economy: A Triple Helix of University-Industry-Government Relations (pp. 1-8). London: Pinter.

European Indicators and Ranking Methodology for University Third Mission - E3M. (2012a). Green paper: fostering and measuring 'third mission in higher education institutions. Recuperado de http://e3mproject.eu/Green\%20paper-p.pdf

European Indicators and Ranking Methodology for University Third Mission - E3M. (2012b). Needs and constraints analysis of the three dimensions of third mission activities. Recuperado de http://e3mproject. eu/Concep-Framework-Third-Mission-Indicator.pdf

European Indicators and Ranking Methodology for University Third Mission - E3M (212c). Conceptual Framework for Third Mission Indicator Definition. Recuperado de http://e3mproject.eu/Concep-Framework-Third-Mission-Indicator.pdf

Extecamp. Catálogo de Cursos - 2017. (2017a). Recuperado de http:// www.extecamp.unicamp.br/catalogo.asp

Extecamp. Relatório de Atividades 2016. (2017b). Recuperado de http://www.extecamp.unicamp.br/documentos-extecamp/relatorios/ extecamp_relatorio_atividades_2016.pdf

Fernández-Larrea, C. M. G. \& González, C. G. R. G. ¿Extensión universitaria, proyección social o tercera misión? Una reflexión necesaria. Congreso Universidad, v. 2, $\mathrm{n}^{\circ}$ 2, 2013, p. 1-11. Recuperado de em http://www.congresouniversidad.cu/revista/index.php/rcu/article/view/482

Giordani, M. C. (1982). História do Mundo Feudal II: civilização. Petrópolis: Vozes.

Göransson, B.; Maharajh, R. \& Schmoch, U. (2009). Introduction: New challenges for universities beyond education and research. Science and Public Policy, 36(2), p. 83-84. https://doi. org/10.3152/030234209X406872
Grau, F. X. (2014). The "Glocal" University. Recuperado de http:// www.guninetwork.org/files/glocal-university_fx_grau_2014.pdf

Haskins, C. H. (2007). The Rise of Universities. New Brunswick (USA) and London (UK): Transaction Publishers.

Grao, J.; Iriarte, M.; Ochoa, C. \& Vieira, M. J. (Coords.). (2014). La Tercera Misión (3M) de las universidades: buenas práticas em la América Latina. México [S.1.]: Imaginarial Editores.

Inova Unicamp. (2018). Indicadores. Recuperado de http://www.inova.unicamp.br/sobre-a-inova/indicadores/

Inova Unicamp. Relatório de Atividades da Agência de Inovação da Inova Unicamp 2016. (2017). Recuperado de https://issuu.com/inovaunicamp4/docs/inv_relativ_2017-low

Instituto Nacional da Propriedade Industrial - INPI. Indicadores de Propriedade Industrial 2018. Recuperado de http://www.inpi.gov.br/ sobre/estatisticas/arquivos/pagina-inicial/indicadores-de-propriedade-industrial-2018_versao_portal.pdf

Inzelt, A., Laredo, P., Sanchez, P., Marian, M., Vigano, F. \& Carayol, N. (2006). Third Mission. In: Schoen A. (Ed.). Strategic Management of University Research Activities, Methodological Guide (pp. 7-19). Lugano: PRIME Project 'Observatory of the European University. Recuperado de http://www.enid-europe.org/PRIME/documents/ OEU_guide.pdf

Kerr, C. (2005). Os usos da Universidade. 15. ed. Brasília: UNB.

Mackinder, H. J. \& Sadler, M. (1891). University extension, past, present, and future. London: Cassell, 1891. Recuperado de https://archive.org/details/extensionunivers00mackrich

Molas-Gallart, J., Salter, A., Patel, P., Scott A., \& Duran, X. (2002). Measuring third stream activities: Final report to the Russell Group of Universities. SPRU, University of Sussex. Recuperado de https://www. academia.edu/532097/Measuring_third_stream_activities

Mora, J. G. \& Vieira, M. J. (Coord.). (2014). Documento de recomendaciones. El fomento de la "Tercera Misión" en las Instituciones de Educación Superior en América Latina. Conclusiones y recomendaciones para los distintos actores. Recuperado de http://recla.org/wp-content/ upl oads/2015/01/Documento-de-Recomendaciones-Vinculaentorno.oct-2014.pdf

Pinheiro, R. (2012). In the Region, for the Region? A comparative study of the institutionalisation of the regional mission of universities (Doctoral dissertation, University of Oslo, Faculty of Educational Sciences). Recuperado de

https://www.academia.edu/5494496/In_the_Region_for_the_Region_A_comparative_study_of_the_institutionalisation_of_the_regional_mission_of_universities_PhD_dissertation_University_of_ Oslo_ 
Ribeiro, D. A universidade necessária. 4. ed. Rio de Janeiro, RJ: Paz e Terra, 1982.

Roessler, I. Duong, S. \& Hachmeister, C. D. (2015). Teaching, Research and more?! Achievements of Universities of Applied Sciences with regard to Society. Centrum für Hochschulentwicklung gGmbH. Recuperado de https://www.che.de/downloads/CHE_AP_183_Third_Mission_at_UAS.pdf

Schoen, A. \& Thèves, J. (2006). OEU strategic Matrix. In: Schoen A. (Ed.). Strategic Management of University Research Activities, Methodological Guide, (pp. 7-20). Lugano: PRIME Project 'Observatory of the European University. Recuperado de http://www.enideurope.org/PRIME/documents/OEU_guide.pdf

Universidade Estadual de Campinas. (2017). Anuário Estatístico 2017: base de dados de 2016. Recuperado de https://www.aeplan.unicamp. br/anuario/2017/anuario2017.pdf
Universidade Estadual de Campinas. (2017). Linha do Tempo. Recuperado de http://www.50anos.unicamp.br/linha-do-tempo

Universidade Estadual de Campinas. (2017). Hospital de Clínicas: ensino e pesquisa. Recuperado de https://www.hc.unicamp.br/node/71

Universidade Estadual de Campinas. (2017). Sobre a PRP. Recuperado de https://www.prp.unicamp.br/pt-br/sobre-a-prp

Universidade Estadual de Campinas. (2017). Saúde. Recuperado de http://www.unicamp.br/unicamp/saude

Welch, E. (1973). The Peripatetic University: Cambridge Local Lectures 1873-1973. London: Cambridge University Press. 